\title{
Variant Typesof Domination in Spinner Graph
}

\author{
Al-Harere M. N. and Athraa T. Breesam \\ Department of Applied Sciences, University of Technology, Baghdad, Iraq \\ 100035@uotechnology.edu.iq \\ A.T.Breesam@gmail.com
}

\begin{abstract}
A new graph, finite, simple, undirected and connected, is introduced named spinner graph, and denoted by $\left(W_{n}\right)^{k}$. This paper includes a study of the domination number (domination, independent domination, total domination, connected domination, restrained domination and, bi-domination) in spinner graph. [DOI: $10.22401 /$ ANJS.00.2.18]
\end{abstract}

Keywords: dominating set, independent dominating set, total dominating set, connected dominating set, restrained domination,bi-dominating set, spinner graph.

Mathematical subject classification: 05C69

\section{Introduction}

We consider a finite undirected and connected simple graph $G=(E, V)$ with a set $V(G)$ of vertices of order $p$, and a set $E(G)$ of edges of size $q$. For a vertex $v \in V(G)$, the degree of a vertex $v$ of any graph $G$ is defined as the number of edges incident on $v$.It is denoted by $\operatorname{deg}(v)$. The minimum and maximum degrees of vertices in $G$ are denoted by $\delta(G)$ and $\Delta(G)$, respectively. The distance $d(v, u)$ between two vertices $v$ and $u$ is the length of a shortest path joining $v$ and $u$, the diameter of $G$ denoted $(\operatorname{diam}(G))$ is the maximum distance among all pairs of vertices in $G$.

The open neighborhood $N(v)$ of $v$ is the set of vertices adjacent to $v$, and the closed neighborhood $N[v]=N(v) \cup\{v\}$. The subgraph of $G$ induced by the vertices in set $D$ is denoted by $G[D]$. For more information see [3].

C. Berge and O. Ore was the first who introduced domination graph $G=$ $(E, V)$ theoretic concept, O. Ore [7] who introduced the expression dominating set and domination number. A set $D \subseteq V$ is a dominating set in $G$ if every vertex in $V-D$ is adjacent to a vertex in $D$, that is $N[D]=$ $V$. The minimum cardinality over all dominating set in $G$ is the domination number of $G$ and is denoted by $\gamma(G)$. Several types of domination parameters have been studied by imposing several conditions on dominating setscan be found in two books written by Haynes et al. [5, 6]. A dominating set $D$ is an independence dominating set in $G$ if $G[D]$ doesn't has edges, the independent domination number of $G$ and is denoted by $\gamma_{i}(G)$. If $G[D]$ has no isolated vertex, then it is called total dominating set in $G$, the total domination number denoted by $\gamma_{t}(G)$. Dis a connected dominating set in $G$, if $G[D]$ is connected. The connected domination number of $G$ denoted by $\gamma_{c}(G)$.A dominating set $D$ is a restrained dominating set where every vertex in $V-D$ is adjacent to a vertex in $D$, as well as, another vertex in $V-D$.The restrained domination number of $G$ denoted by $\gamma_{r}(G)$ [4].A dominating set $D \subset V(G)$ is a bi-dominating set in $G$, if every vertex $v$ in $D$ dominates exactly two vertices in $V-D$, the minimum cardinality of bi-dominating set is denoted by $\gamma_{b i}(G)[1,2]$.

Dominating sets can be used in modeling social networks and studying the dynamics of relations among numerous people in different fields. A social network is a social structure made of people, connected by one or more types of interdependency. The choice of initial sets of target people is an important problem in the theory of social networks. Social networks are modeled in terms of graph theory and it was shown that some of these sets can be found by using the properties of dominating sets in graphs.

Here, a new model of graph called spinner is initiated. The number of vertices and edges of spinner are calculated and since spinner graph, is constructed of wheel graphs, so the number of these wheels is also 
calculated. This graph manly resemble to hierarchy of an enterprise, where the center vertex represent the highest level of the hierarchy (CEO), who dominates other helpers (core graph vertices excluding the center), who convey orders to other executives (centers of connected wheel graphs).This can represent Local Area Network (LAN), the Local Area Network (LAN) are networks used in small businesses and universities to facilitate the rapid transfer of information and information between departments within the scope of a limited deployment for example (the work of the office-internal institutions-limited companies). Spinner graph is not only for (LAN) but also for Wide Area Network (WAN) by rising the value of $(\mathrm{k})$ in spinner graph, where (WAN) represents all networks that span a wide geographic area and may be a city, a continent or the whole world. There are large private networks, for. Each wheel can be a country connected with other countries with a leader (the center vertex in each wheel). In this paper different formulas for domination (domination, independent domination, total domination, connected domination, bidomination) are determined in spinner graph. Finally, these domination types have been compared.

Theorem1.1. [2] For a wheel graph $W_{n}$, $n \geq 3$ and $n \neq 5, \gamma_{b i}\left(W_{n}\right)=2\left\lceil\frac{n}{4}\right\rceil$.

\section{Main Results}

Definition 2.1. (Spinner graph). A spinner graph $\left(W_{n}\right)^{k}, k \geq 2$ is constructed recursively from a sequence of wheel graphs ( $a$ wheel $W_{n}$ is the graph $C_{n}+K_{1}$ ) by augmenting $n$ copies of wheel graph to then vertices of $W_{n}$ (The graph $W_{n}$ has $n+1$ vertices and 2 nedges is the core of spinner graph also will be known as layer one) by making a common vertex between the joined graphs (not a center vertex), to form spinner graph $\left(W_{n}\right)^{2}$ called layer two. Similarly, we augment $W_{n}$ to each of the $n-1$ vertices on each wheel of $\left(W_{n}\right)^{2}$ except the center vertex in each wheeland except the common vertices with the layer one to form $\left(W_{n}\right)^{3}$ (layer three), such that in layer $i, 3 \leq i \leq k$ the augmenting of wheel graphs only for vertices of wheels in layer $i-1$ and so on (see Figure 1).

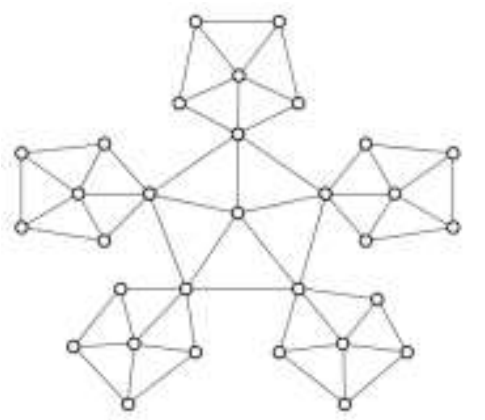

Figure 1: Spinner $\operatorname{graph}\left(W_{5}\right)^{2}$.

Note 2.2. Number of vertices and edges in $W_{n}$ are equal to $n+1$ and $2 n$, respectively.

Lemma 2.3. The number of wheel graphs in spinner graph for $n \geq 3$ is:

$U=1+n\left(\sum_{i=0}^{k-2}(n-1)^{i}\right), k \geq 2$

Proof. Firstly, it is obvious that the number of wheels is equal to $1+n$ when $k=2$, since the core of spinner graph contains one wheel and layer two contains $n$ wheels.

Secondly, if $k \geq 3$, by induction method let us assume that the number of wheels $U$ is true for $s=k-1$, which mean that the number of wheels in this subgraph $\left(W_{n}\right)^{k-1}$ is $1+$ $n\left(\sum_{i=0}^{k-3}(n-1)^{i}\right)$. In the next step the layer $k$ is added to the previous subgraph $\left(W_{n}\right)^{k-1}$. The number of vertices (excluding the center) in layer $k-1$, which is not common with the vertices of the previous wheels is $n(n-$ $1)^{k-2}$, so by Definition 2.1 of spinner graph, one can add $n(n-1)^{k-2}$ wheel graphsto obtain the number of wheel graphs inlayer. Thus, the number of wheels in the graph is:

$1+n\left(\sum_{i=0}^{k-3}(n-1)^{i}\right)+n(n-1)^{k-2}=1+$ $n\left(\sum_{i=0}^{k-2}(n-1)^{i}\right)$.

Theorem 2.4. Spinner graph has $p$ and $q$ edges vertices for $n \geq 3$, where:

(i) $p=1+n+n^{2}\left(\sum_{i=0}^{k-2}(n-1)^{i}\right), k \geq 2$,

(ii) $q=2 n\left(1+n\left(\sum_{i=0}^{k-2}(n-1)^{i}\right)\right)$

\section{Proof.}

(i) The core of spinner graph is a wheel containing $n+1$ vertices, and each of the other wheels increase the number of vertices of the spinner by $n$ vertices. Thus, the number of vertices can be obtained by multiplying the number of wheels $(U)$, which is computed 
inLemma 2.3andthen multiplied by $n$ and then adding one to the previous summation, which means:

$n\left(1+n\left(\sum_{i=0}^{k-2}(n-1)^{i}\right)\right)+1=1+n+$ $n^{2}\left(\sum_{i=0}^{k-2}(n-1)^{i}\right)$, when $k \geq 2$

(ii) The number of edges in a wheel graph of order $n+1$ is $2 n$ referring to (Note 2.2) and all the wheels in spinner graph have no common edges between them, then:

$q=2 n U=2 n\left(1+n\left(\sum_{i=0}^{k-2}(n-1)^{i}\right)\right)$

for all $k \geq 2$.

Remark 2.5. Let $v$ be a vertex which represent the common vertex between any two wheel graphs.So, $\operatorname{deg}(v)=6$ and let the vertex $u$ be a vertex representing the center of any wheel has $\operatorname{deg}(u)=n$. Thus, $\Delta(G)=$ $\operatorname{deg}(v)$ if $n \leq 5, \Delta(G)=\operatorname{deg}(u)=$ $\operatorname{deg}(v)=6$ if $n=6, \Delta(G)=\operatorname{deg}(u)$ if $n \geq 7, \quad \delta(G)=3$ if $n \geq 4$ and $\delta(G)=$ $\operatorname{deg}(u)$ if $n=3$.

Remark 2.6. In spinner graph, we labelthe vertices in this paper as follows. The vertices of core (wheel $W_{n}$ ) in spinner graph are labeled such that the center vertex of $W_{n}$ be $v_{1}$, then the remaining $n$ vertices are labeled with clockwise $\left\{v_{2}, \ldots, v_{n+1}\right\}$. Then, when $k=2$ the vertices are labeled starting with common vertices in the last step of the less label $\left(v_{2}\right)$ and the remaining vertices are labeled in this wheel starting with the center and then the remaining vertices clockwise $\left\{v_{n+2}, \ldots, v_{\left|V\left(W_{n}\right)^{2}\right|}\right\}$. So, we complete labeling clockwise in each wheel with noted that starting in center and then the $n-1$ vertices for each wheel and so on (see Figure 2).

\section{Dominating Set in Spinner Graph}

Theorem 3.1. For a spinner graph $\left(W_{3}\right)^{k}$, $k \geq 2, \gamma\left(W_{3}\right)^{k}=2^{k}-1$.

Proof. Let $D \subseteq V\left(\left(W_{n}\right)^{k}\right)$. To construct the minimum dominating set in spinner graph, we must first choose one vertex from each wheel in layer $k$ (terminal wheel) in the set $D$. The best candidate vertex is a common vertex with the previous wheel in layer $k-1$, since this vertex has the maximum neighborhood. The set of those vertices dominate all vertices in $k$ and $k-1$ layers and the number of these vertices is the minimum because if one vertex is deleted from $D$ we cannot dominate the vertices of wheel, which this vertex belong to it. In the same manner the common vertices between the wheels in $k-2$ and $k-3$ layers are chosen to dominate all the vertices of the wheels in this scope. By continuing this procedure till reaching the core of this graph leads to getting the following set:

$$
\begin{aligned}
D_{1}= & \left\{v_{3 i}, v_{3 i+1}, i=3\left(2^{j-3}\right)-\right. \\
& \left.1, \ldots, 3\left(2^{j-2}\right)-2, \forall j=4,6, \ldots, k\right\}
\end{aligned}
$$

when $k$ is an even number and:

$$
\begin{aligned}
& D_{1}=\left\{v_{3 i}, v_{3 i+1}, i=3\left(2^{j-3}\right)-\right. \\
& \left.1, \ldots, 3\left(2^{j-2}\right)-2 \forall j=3,5, \ldots, k\right\}
\end{aligned}
$$

when $k$ is an odd number, which is the minimum dominating set for all previous layers.The remaining vertices thatare not dominated by the vertices in the previous layers will be dominated as follows:

(i) If $k$ is even, then the remaining vertices that are not dominated by the previous layer are the verticesof layertwo $(k=2)$ and the core (layer one) of spinner. Similarly,the vertices are chosen to dominate the vertices in this layer. It's clear that the set of these vertices is $D_{2}=\left\{v_{2}, v_{3}, v_{4}\right\}$.

(ii) If $k$ is odd, then the last stage, which are reached without domination contains the vertices of the core of spinner $\left(W_{3}\right)$. Thus, the center of this wheel dominates all vertices in it, which means that, $D_{2}=\left\{v_{1}\right\}$ (see Figure 2).

Therefore, for all cases above the dominating set with minimum cardinality is $D=D_{1} \cup$ $D_{2}$.



Figure 2: Minimum dominating set in $\left(W_{3}\right)^{3}$. 
Theorem 3.2. For $n \geq 4$, spinner $\operatorname{graph}\left(W_{n}\right)^{k}, k \geq 2$ has:

$\gamma\left(W_{n}\right)^{k}=1+n\left(\sum_{i=0}^{k-2}(n-1)^{i}\right)$

Proof. Let $D \subseteq V\left(\left(W_{n}\right)^{k}\right)$, where the vertices in $D$ represent all centers of wheel graphs in spinner, so $|D|=U$, where $U$ represent the number of wheels in spinner graph being is calculated in Lemma 2.3.

It is clear that $D$ is a minimal dominating set, since the center of each wheel dominates all vertices in this wheel. Also, if one vertex is deleted from $D$ say $v$, then there is at least one vertex $(v)$, which is not dominated by set $D-\{v\}$. These vertices belong to the wheel (itscenter $v$ ) which is deleted from $D$.

Now, to prove $D$ has minimum cardinality, there are two steps to discuss as follows.

(i) The vertices of any wheel (specifically the center vertex) cannot be dominated by any vertices that do not belong to the same wheel. So, there is at least one vertex from each wheel in the dominating set $D$.

(ii) If the vertex chosen in (i) say $u$ is not the center, then the candidate vertex is the common vertex with the other wheelssay $w$. Since, $n \geq 4$, then $\operatorname{diam}\left(W_{n}\right)=2$ and so there is at least one vertex from this wheel is not dominated by the vertex $w$. Therefore two wheel graphs cannot be dominated by one vertex. Thus:

$$
\begin{aligned}
\gamma\left(W_{n}\right)^{k} & =|D| \\
& =U \\
& =1+n\left(\sum_{i=0}^{k-2}(n-1)^{i}\right), k \geq 2
\end{aligned}
$$

Theorem 3.3. For spinner graph $\left(W_{n}\right)^{k}, k \geq$ 2

(i) $\gamma_{i}\left(W_{3}\right)^{k}=2^{k}-1$, and,

(ii) $\gamma_{i}\left(W_{n}\right)^{k}=U=1+n\left(\sum_{i=0}^{k-2}(n-\right.$

1) ${ }^{i}$ ) if $k \geq 2$.

Proof. (i) Let $D \subseteq V\left(\left(W_{3}\right)^{k}\right)$. Two cases are gotten:

Case1. If $k$ is even, let the set $D=D_{1} \cup D_{2}$ where,

$D_{1}=\left\{v_{2}, v_{8}, v_{11}\right\}$

$D_{2}=\left\{v_{3 i+1}, v_{2(3 i+1)}, i=3\left(2^{j-3}\right)-\right.$

$\left.1, \ldots, 3\left(2^{j-2}\right)-2 \forall j=4,6, \ldots, k\right\}$,

If $k=2$, then set $D_{2}=\phi$.

It's clear that $D_{1}$ is a minimum independent dominating set when $k=2$. So, when $k \geq 4$ and according to Remark 2.5, the common vertices have the maximum neighbors in
spinner.However, these vertices are adjacent within the same wheel so common vertex and center vertex are chosen clockwise and alternating as in set $D_{2}$.

Case2. If $k$ is odd, let the set $D=D_{1} \cup D_{2}$, where:

$$
\begin{aligned}
D_{1}= & \left\{v_{1}\right\}, \\
D_{2}= & \left\{v_{3 i+1}, v_{2(3 i+1)}, i=3\left(2^{j-3}\right)-\right. \\
& \left.1, \ldots, 3\left(2^{j-2}\right)-2 \forall j=3,5, \ldots, k\right\}
\end{aligned}
$$

The same technique can be used for the proof in Case 1.

In the two cases $D$ is the minimum independent dominating set.

(ii)For $n \geq 4$, by Theorem 3.2 the dominating set consisted ofthe center vertex of each wheel, since these vertices are independent. Thus, the minimum dominating set in Theorem 3.2 isthe minimum independent dominating set.

Theorem 3.4. A spinner graph $\left(W_{3}\right)^{k}, k \geq 2$, has:

$\gamma_{t}\left(W_{3}\right)^{k}=\left\{\begin{array}{c}2^{k}-1 \text { if } k \text { is even } \\ 2^{k} \text { if } k \text { is odd }\end{array}\right.$

Proof.Let $D$ be a set such that $D \subseteq$ $V\left(\left(W_{3}\right)^{k}\right)$, then according to the value of $k$ two cases are obtained

Case1. If $k$ is even

The dominating set in Theorem 3.1 (Case1) represents the total dominating set in $\left(W_{3}\right)^{k}$.

Case2. If $k$ is odd

The dominating set in Theorem 3.1 (Case2) represents the total dominating setafter adding a vertex to set $D_{1}$ to satisfythe condition of the total domination, so $D_{1}=\left\{v_{1}, v_{2}\right\}$. Hence, the result can be obtained.

Theorem 3.5. A spinner graph $\left(W_{n}\right)^{k}, n \geq$ $4, k \geq 2$, has:

$$
\gamma_{t}\left(W_{n}\right)^{k}=\left\{\begin{array}{cc}
2 n & \text { if } k=2 \\
n+2 \sum_{i=2}^{\frac{k}{2}} n(n-1)^{2 i} & \text { if } k \text { is even } \\
2+2 \sum_{i=2}^{\frac{k+1}{2}} n(n-1)^{2 i-1} & \text { if } k \text { is odd }
\end{array}\right.
$$

Proof. To prove this theorem we use the number of wheels $U$ calculated by Lemma 2.3 , there are three cases based on the number of layers $(k)$ in spinner as follows. Let $\subseteq V\left(\left(W_{n}\right)^{k}\right)$.

Case 1. If $k=2$, to dominate the wheel graphs in layer two the central vertices are chosen in set $D$ to dominate each wheel with 
the common vertices to be a total dominating set. So $D$ dominates all vertices in spinner (core and layer two). Additionally, we cannot lose any vertex from this set because the totaldomination of one of the wheel graphs may be lost. Therefore, the number of these vertices is the minimum cardinality of all total dominating set.Since the number of central vertices is equal to the number of wheels $U$ Lemma 2.3. Hence:

$$
\begin{aligned}
\gamma_{t}\left(\left(W_{n}\right)^{2}\right) & =2(U-1) \\
& =2(1+n-1) \\
& =2 n
\end{aligned}
$$

Thus, the result is obtained.

Case 2. If $k$ is even and greater than or equal to four, then firstly, the last layer is the one we start with, going towards the core. So, the center of each wheel is chosen in this layer. Again,in the same manner in (Case1), another vertex from each wheel must be chosen to totallydominate this wheel. In the same way in (Case1) the set of these vertices is the minimum cardinality and it is a total dominating set. Additionally, these vertices totally dominate all wheel graphs in the last two layers ( $k$ and $k-1$ ). According to Lemma 2.3, the number of wheels in the last layer $k$ is $n(n-1)^{k-2}$, so the minimum number of vertices that totally dominate the last two layers is $2 n(n-1)^{k-2}$. Again, in the same manner the next two layers are totally dominated and so on, until reaching layer one and by using the proof in (Case1) one can get the result.

Case 3. If $k$ is odd and greater than or equal to three, in the same way in (Case 2), the minimum number of vertices are chosen as total domination vertices. The only difference from the previous manner is the last step, where we reach the core only two vertices are added to totally dominate the core. Thus, we get the result (see Figure 3).

By combining the results from Cases 1, 2, and 3 , one can get the required result.



Figure 3: Minimumtotal dominating set in $\left(W_{4}\right)^{3}$.

Theorem 3.6. A spinner graph $\left(W_{n}\right)^{k}, n \geq$ $3, k \geq 2$, has

$$
=\left\{\begin{array}{l}
\gamma_{c}\left(W_{n}\right)^{k} \\
n+\left(n^{2}-n\right)\left(\sum_{i=0}^{k-3}(n-1)^{i}\right), \text { if } n=3 \\
n+n)\left(\sum_{i=0}^{k-3}(n-1)^{i}\right)+n(n-1)^{k-2}, \text { if } n \geq 4
\end{array}\right.
$$

Proof. To have a connected domination on the spinner, all the common vertices are chosen. All the vertices that are locatedon the circle in any internal wheel will have to belong to the connected dominating set $(D)$. It is clear that these vertices are connected and dominate all vertices of the subgraph $\left(W_{n}\right)^{k-1}$. Additionally, the set of these vertices has a minimum cardinality, because if any vertex is deleted from $D$ then $G[D]$ will be a disconnected subgraph. Thus, the cardinal of this set is $|D|=1+n+$ $n^{2}\left(\sum_{i=0}^{k-3}(n-1)^{i}\right)-\left(1+n\left(\sum_{i=0}^{k-3}(n-1)^{i}\right)\right)$ (by Theorem 2.4 and Lemma 2.3). Now, there 
are two cases depending on the value of $n$ as follows.

(i) If $n=3$, then the set $D$ is the minimum dominating set of all vertices of graph.

(ii) If $n \geq 4$, then the remaining vertices, that are not a connected domination by the set $D$, are the vertices in layer $k$ such that at least one vertex from each wheel in layer $k$ is not dominated by $D$. Thus, the center of each wheel in the layer $k$ is added to set $D$ to get a connected dominating set with minimum cardinality. Also, it is clear that the number of the central vertices in each layer is equal to the number of wheel graphs in the same layer sothis number is equal to $n(n-1)^{k-2}$ (by Lemma 2.3). Thus, the result is proved.

Theorem 3.7. A spinner graph $\left(W_{n}\right)^{k}, k \geq$ 2 has $\gamma_{r}\left(W_{n}\right)^{k}=\gamma\left(W_{n}\right)^{k}$

Proof. Let $D$ be the dominating set in Theorems 3.1 and 3.2. From the definition of restrained dominating set, it is clear thatevery vertexbeginnot in dominating set is adjacent to a vertex in $D$ and to at least two vertices in $V\left(\left(W_{n}\right)^{k}\right)-D$.Therefore, $D$ is the restrained dominating set and $\gamma_{r}\left(W_{n}\right)^{k}=\gamma\left(W_{n}\right)^{k}$.

Theorem3.8. A spinner graph $\left(W_{n}\right)^{k}$ doesn't have bi-dominating set when $n=3,6$.

Proof. To establish the bi-dominating set, there are three methods tochoose the vertices of this set as follows:

(i) If we take all common vertices in a graph, so these vertices are bi-dominating to subgraph $\left(W_{n}\right)^{k-1}$. Now, the vertices which are not bi-dominated are located in layer $k$, thus there are three ways for selecting the rest of the vertices.

(a) If no vertex is selected, then the common vertices dominate four vertices and this is a contradiction with the definition of bidominating set.

(b) If one vertex is selected, again the common vertex dominates three vertices and this is alsoa contradiction.

(c) If two or three vertices are selected, then these vertices do not dominate two vertices in $V-D$. Again this is a contradiction.

Therefore, we cannot establish the dominating set by this way.

(ii) If we take only center of wheel graphs, then it is obvious this set is not a dominating set. This is because every center vertex dominates three vertices and this is a contradiction.

(iii) If we mixthe two ways mentioned in (i) and (ii), i.e., selection the center and common vertices, then there is at least one wheel with contains center and common vertices. Now, the center vertex in this wheel dominates the other vertices in the same wheel. Thus, we cannot select these vertices in a bi-dominating set, but the degree of the common vertices is 6 by Remark (2.5). Therefore, we must select three vertices in $D$ from wheel graphs such that they have a common vertex and this is a contradiction with the definition of bidominating set.

Thus, there is no way to establish the bidominating set and the proof is completed.

Theorem 3.9. For $n \geq 4, n \neq 6, k \geq 2$ the bi-domination number of spinner graph is:

$\gamma_{b i}\left(\left(W_{n}\right)^{k}\right)=2 n(n-1)^{k-2}\left\lceil\frac{n-1}{4}\right\rceil+n+$ $\left(n^{2}-n\right)\left(\sum_{i=0}^{k-3}(n-1)^{i}\right)$

when $k=2, \sum_{i=0}^{k-3}(n-1)^{i}=0$.

Proof. Let $D$ be a set such that $D \subset$ $V\left(\left(W_{n}\right)^{k}\right)$. Now, let all the common vertices in spinner graphbelong to set $D$. Thus, by Theorem 3.6 the number of these vertices is $n+\left(n^{2}-n\right)\left(\sum_{i=0}^{k-3}(n-1)^{i}\right)$. The set $D$ is a bi-dominating set in spinner graph since each common vertex is adjacent to two central vertices (see Figure 4) except the vertices on the cycle of each wheel graph in layer $k$. So, the remaining vertices that are not bidominated by set $D$ belong to the layerk. Note that the common vertex from each wheel on the layer $k$ belongs to set $D$. Also, the vertices that are adjacent to the common vertex (except center vertex) in the cycle of each wheel in layer $k$ must belong to set $D$ to get a bi-dominating set. Thus, by booking the common vertices in set $D$ and applying Theorem1.1, to get the number of vertices that dominate the remaining vertices as a bidomination foreach wheel except the booked vertex, is $2\left\lceil\frac{n-1}{4}\right\rceil$ (as shown in Figure 4). Thus, the new vertices belong to $D$ in layer $k$ is $2 n(n-1)^{k-2}\left\lceil\frac{n-1}{4}\right\rceil$. Therefore, the result is obtained. 


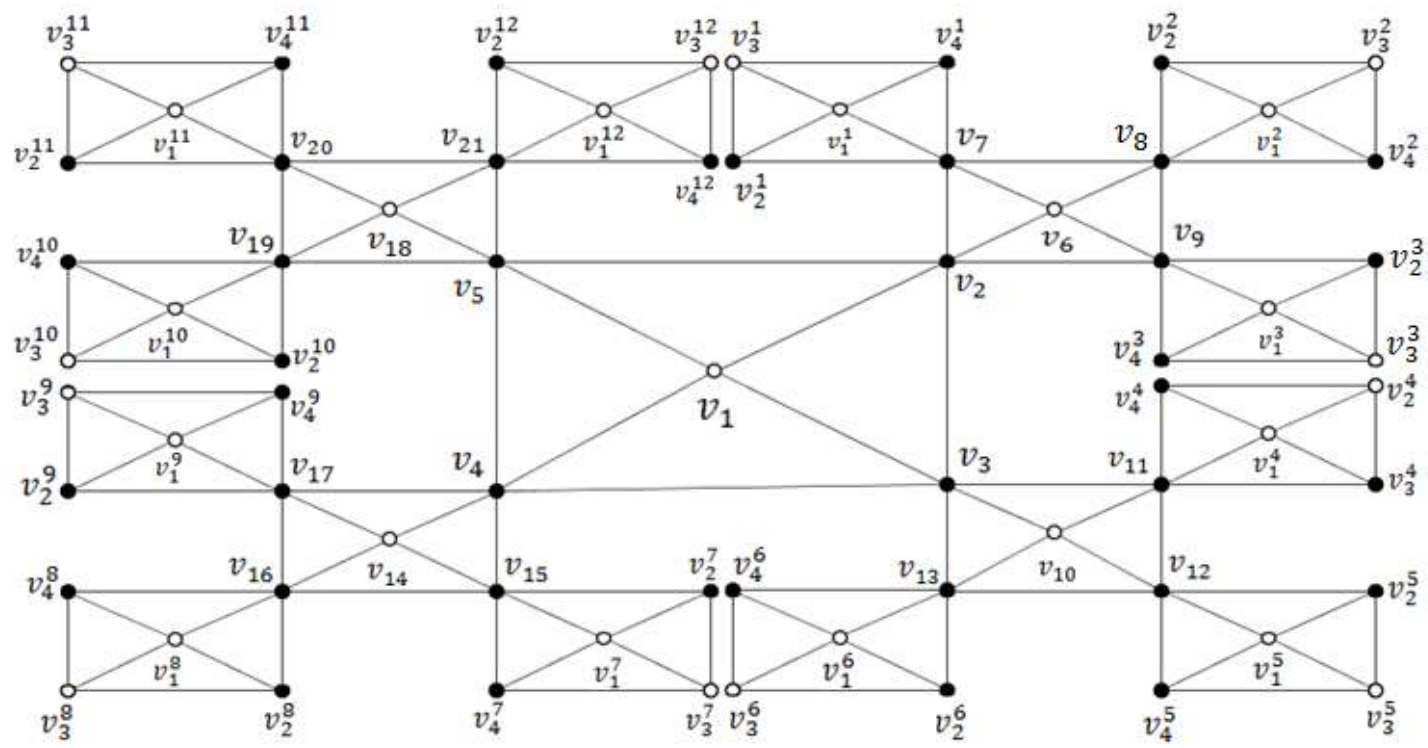

Figure 4: Minimum bi-dominating set in $\left(W_{4}\right)^{3}$.

\section{Conclusion}

In this paper, some types of domination (domination, independent domination, total domination, connected domination, restrained domination and bi-domination) are studied in spinner graph by finding the domination number and dominating set for each type, the comparison between these types for spinner graph is as follows:

$\gamma\left(\left(W_{n}\right)^{k}\right)=\gamma_{r}\left(\left(W_{n}\right)^{k}\right) \leq \gamma_{i}\left(\left(W_{n}\right)^{k}\right) \leq$ $\gamma_{t}\left(\left(W_{n}\right)^{k}\right)<\gamma_{c}\left(\left(W_{n}\right)^{k}\right)<\gamma_{b i}\left(\left(W_{n}\right)^{k}\right)$

\section{References}

[1] M. N. Al-Harere and Athraa T. Breesam, Bi-domination in graphs, reprint.

[2] M. N. Al-Harere and Athraa T. Breesam, Inverse bi-domination in graphs, accepted inInternational Journal of Pure and Applied Mathematics, (2017).

[3] Gary Chartrand, Linda Lesniak, Ping Zhang, Graphs \& Digraphs, Sixth Edition, Taylor \& Francis Group, (2015).

[4] Gayla S. Domke, Johannes H. Hattingh, Stephen T. Hedetniemi, Renu C. Laskar, Lisa R. Markus, Restrained domination in graphs, Elsevier Science, (1999).

[5] F. Harary, Graph Theory, AddisonWesley, Reading Mass, (1969).

[6] T. W. Haynes, S.T. Hedetniemi and P. J. Slater, Fundamentals of domination ingraphs, Marcel Dekker, Inc., New York (1998).
[7] O. Ore. Theory of graphs, American Mathematical Society, Provedence, R.I., (1962). 